\title{
Uso rutinario del montaje lateral (side-docking) en cirugía robótica ginecológica: estudio de factibilidad
}

\author{
Iván Rojas $T^{1}{ }^{1}$, Demetrio Larraín de la C. ${ }^{1}$, Francesca Marengo ${ }^{1}$, Felipe González L. ${ }^{1}$, \\ Jaime Prado A. ${ }^{1}$, Hans Buckel G. ${ }^{1}$ \\ ${ }^{1}$ Servicio de Obstetricia y Ginecología, Clínica Santa María. Santiago, Chile.
}

\section{RESUMEN}

Antecedentes: La mayoría de los estudios publicados en cirugía robótica ginecológica han utilizado el montaje central, con el robot entre las piernas de la paciente. Una desventaja importante de esta técnica es el limitado acceso vaginal. Recientemente, el uso del montaje lateral (robot en $45^{\circ}$ respecto a la mesa operatoria) ha permitido obviar esta dificultad. Objetivo: Demostrar la factibilidad del montaje lateral desde el inicio del programa de cirugía robótica en nuestra institución. Método: Estudio de cohorte prospectivo de 22 pacientes consecutivas que optaron por cirugía robótica. Todas las cirugías se realizaron con montaje lateral, recolectando datos demográficos y perioperatorios en cada caso. Resultados: No hubo conversiones a laparotomía o cirugía vaginal. La edad media fue 46 años y el índice de masa corporal medio fue de $26 \pm 5$ $\mathrm{kg} / \mathrm{m}^{2}$. Los tiempos medios de trócares y montaje fueron $12 \pm 6$ y $12 \pm 4$ minutos, respectivamente. El tiempo de consola medio fue $111 \pm 60$ minutos. Al comparar nuestros primeros 10 casos con el resto, encontramos una disminución significativa en el tiempo de montaje $(p=0,03)$ y el tiempo de consola $(p=0,02)$. Los procedimientos realizados fueron los siguientes: histerectomía $(n=18)$, promonto fijación $(n=1)$, anexectomía $(n=1)$, resección endometriosis $(n=1)$, linfadenectomía pelviana $(n=4)$, linfadenectomía lumbo-aórtica $(n=1)$. Dos pacientes experimentaron complicaciones: una lesión vesical y una perforación intestinal. La estadía hospitalaria media fue 36 horas. Conclusión: El montaje lateral es fácil de adoptar y proporciona un acceso vaginal más adecuado, facilitando la exposición y la extracción de especímenes durante la cirugía robótica ginecológica.

\section{PALABRAS CLAVE: Cirugía robótica, cirugía robótica ginecológica, montaje lateral, curva de aprendizaje}

\section{SUMMARY}

Background: Most of studies on robotic gynaecologic surgery have used traditional docking, with the robot located between the patient's legs. A significant limitation is the limited vaginal access. Recently, side docking with the robot docked at $45^{\circ}$ angle to the lower torso has been described. Objective: To report our experience using the side docking since the beginning of our robotic program. Method: 22 consecutive patients who underwent robotic procedures for gynaecological diseases were included. All surgeries were performed using the side-docking. Data collected included demographics, surgical procedures, operative time, complications, conversion rate and hospital stay. Results: All procedures were completed robotically with no conversion. Mean age was 46 years. Mean BMI was $26 \pm 5 \mathrm{~kg} / \mathrm{m}^{2}$. Mean trocar time was $12 \pm 6$ minutes and the mean docking time was $12 \pm 4$ minutes. Mean operative time was $111 \pm 60$ minutes. When our first 10 cases were compared with the others we found a statistically significant reduction in docking time $(p=0.03)$ 
and console time $(p=0.02)$. Surgical procedures were the following: hysterectomy $(n=18)$, sacrocolpopexy $(n=1)$, annexectomy $(n=1)$, endometriosis resection $(n=1)$, pelvic lymphadenectomy $(n=4)$, aortic lymphadenectomy $(n=1)$. Two complications occurred: a bladder injury and a bowel perforation. Mean hospital stay was 36 hours. Conclusion: Side-docking is easy to learn and improve vaginal access, reduce assistant's fatigue and facilitate specimen retrieval.

\section{KEY WORDS: Robotic surgery, gynaecologic robotic surgery, side docking, learning curve}

\section{INTRODUCCIÓN}

La introducción de la cirugía robótica en ginecología ha contribuido a superar algunas limitaciones de la laparoscopia convencional, manteniendo todas las ventajas de la cirugía mínimamente invasiva. La tecnología robótica permite al cirujano visualizar la cavidad abdominal en tres dimensiones y operar con mayor destreza y precisión comparado con la cirugía laparoscópica, debido al uso de instrumentos articulados, los cuales permiten un mayor rango de movimientos, y la filtración del temblor natural de la mano humana (1). El sistema quirúrgico robótico consiste en una consola ergonómica para el cirujano, una torre endoscópica y un carro móvil con tres o cuatro brazos robóticos que se instala próximo a la paciente en diferentes ángulos (1). En cirugía ginecológica este carro es tradicionalmente montado entre las piernas de la paciente (montaje clásico), con el fin de maximizar la maniobrabilidad de los instrumentos en la pelvis, mantener la simetría de los trocares y minimizar la probabilidad de colisión de los brazos robóticos (2). Sin embargo, uno de los principales problemas de esta forma de montaje es el limitado acceso vaginal, muy importante en cirugía laparoscópica ginecológica $(2,3)$. Recientemente, el montaje lateral del carro robótico parece ser una alternativa interesante para obviar este problema, aunque la experiencia clínica publicada al respecto es limitada (4-6).

El objetivo de este estudio es demostrar la factibilidad del montaje lateral en cirugía robótica ginecológica en nuestro medio, reportando nuestra experiencia desde el inicio del programa de desarrollo de esta cirugía.

\section{PACIENTES Y MÉTODOS}

En noviembre de 2011 se inicia un estudio de cohorte prospectivo que incluye a todas las mujeres con indicación quirúrgica, las cuales optaron por la cirugía robótica. Los criterios de exclusión definidos corresponden a la presencia de cualquier contraindicación anestésica para cirugía laparoscópica estándar y la sospecha de cáncer ginecológico avanzado.

El sistema quirúrgico utilizado fue Da Vinci $\mathrm{Si}$, de segunda generación, con 4 brazos (Intuitive Surgical Inc., Sunnyvale, CA, USA). Las pacientes fueron colocadas en posición de litotomía con los brazos a lo largo el cuerpo. La anestesia fue general. La colocación de sonda Foley previo a la cirugía y el uso de manipulador uterino fue de uso rutinario. El neumoperitoneo se logró mediante la inserción de aguja de Veress a nivel umbilical o en el hipocondrio izquierdo, según los antecedentes de laparotomías previas, siguiendo las normas y principios de seguridad descritos para laparoscopia ginecológica (7). Después de la creación del neumoperitoneo se utilizaron 4 o 5 trocares abdominales: un trocar de $12 \mathrm{~mm}$ en o sobre el ombligo para la cámara, 2 o 3 puertos laterales de $8 \mathrm{~mm}$ para los brazos robóticos y un trocar de $12 \mathrm{~mm}$ para el primer asistente (Figura 1). Las funciones de este último son la aspiración/irrigación, la introducción de suturas y la extracción de tejidos. Un segundo asistente, situado entre las piernas de la paciente, estuvo presente en todas las cirugías con el fin de manejar el manipulador uterino, optimizando la exposición de los órganos pélvicos. En todos los casos, el carro robótico fue aproximado a la paciente utilizando el montaje lateral, situando el robot en un ángulo de aproximadamente $45^{\circ}$ con respecto a la mesa operatoria, por fuera de la cadera izquierda de la paciente (6) (Figura 2). Los datos fueron recolectados en forma prospectiva para cada caso e incluyeron datos demográficos, índice de masa corporal, antecedente de laparotomía, estadía hospitalaria y complicaciones. Otros datos, comúnmente utilizados en cirugía robótica se registraron siguiendo las definiciones que se muestran en la Tabla I. El análisis estadístico se realizó utilizando el software Epilnfo versión 3.5.1, considerando $p<0,05$ como significativo. 


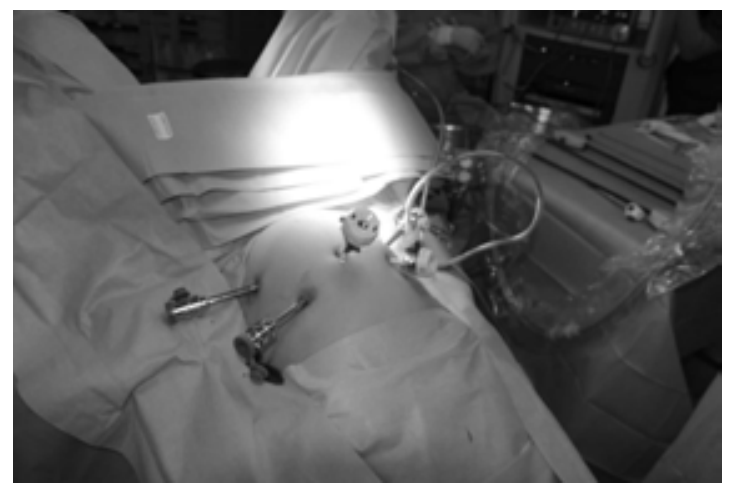

Figura 1. Localización de los trocares utilizados en cirugía robótica ginecológica. Nótese que la distancia entre los trocares debe ser al menos de $8 \mathrm{~cm}$, con el fin de evitar colisión de los brazos y favorecer la ergonomía.

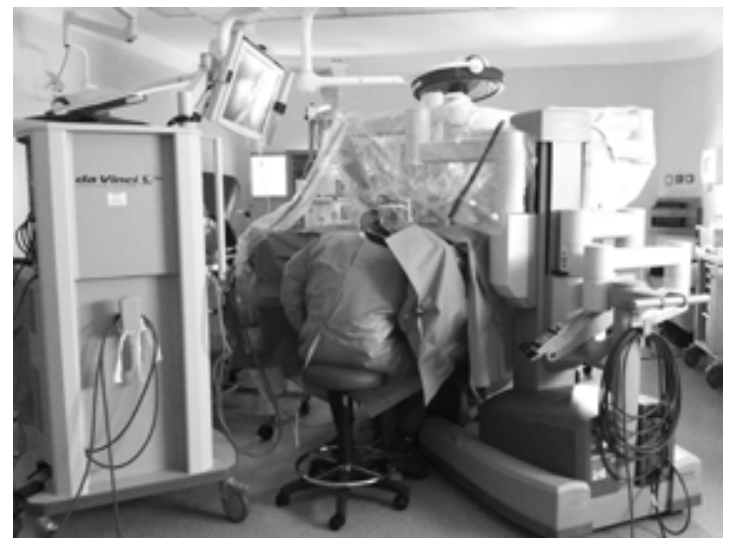

Figura 2. Disposición de la torre endoscópica y el carro robótico utilizando el montaje lateral. Nótese el acceso vaginal y el rango de movilidad disponible para el segundo asistente.

\section{RESULTADOS}

Desde la introducción de la cirugía ginecológica robótica en nuestra institución hemos operado 22 pacientes, las cuales constituyen el grupo de estudio. La edad media fue de 46 años (rango: 30-65 años). El índice de masa corporal medio fue $26 \pm 5$ $\mathrm{kg} / \mathrm{m}^{2}$ (rango: $20-38 \mathrm{~kg} / \mathrm{m}^{2}$ ). El $74 \%$ de las pacientes tenían antecedentes de laparotomías previas, cesáreas en su mayoría. Las indicaciones de la cirugías fueron las siguientes: adenomiosis uterina $(n=6)$, miomatosis uterina sintomática $(n=5)$, sangrado uterino anormal $(n=4)$, cáncer de endometrio $(n=4)$, cáncer de ovario $(n=1)$, prolapso genital $(n=1)$ y endometriosis profunda $(n=1)$.

Los procedimientos realizados se resumen en la Tabla II. No se registraron conversiones a laparotomía o cirugía vaginal. El tiempo medio de instalación de trocares fue de $12 \pm 6$ minutos (rango: 5-37 minutos), el tiempo medio de montaje (acoplamiento de los brazos robóticos a la cámara y a los trocares) fue de $12 \pm 4$ minutos (rango: 4-23 minutos) y el tiempo medio de consola (desarrollo de la cirugía propiamente tal) fue de $111 \pm 60$ minutos (rango: 31-250 minutos) para toda la serie. La curva del tiempo de consola mostró un punto de inflexión en el caso \# 10. Por esta razón comparamos los 10 primeros casos con los restantes 12 casos, encontrándose una diferencia estadísticamente significativa en el tiempo de medio de consola entre los 2 grupos $(143 \pm 68$ minutos vs $85 \pm 36$ minutos, $\mathrm{p}<0,02)$.

Al considerar sólo las histerectomías, el tiempo medio de consola fue de $101 \pm 58$ minutos (rango: 31-250 minutos). En las primeros 10 histerectomías, el tiempo medio de consola fue de $122 \pm 68$ minutos y en los siguientes 8 casos $75 \pm 26$ minutos, diferencia no significativa.

\section{Tabla I}

\section{DEFINICIONES Y PARÁMETROS COMÚNMENTE UTILIZADOS EN CIRUGÍA ROBÓTICA}

Tiempo de trocares

Tiempo de montaje

Tiempo de consola
Desde incisión para Verres. Incluye creación neumoperitoneo, incisiones e introducción de trocares robóticos y auxiliares. Incluye adherenciolisis previa a instalación trocares robóticos de ser necesaria.

Desde el avance del carro robótico a la paciente, hasta la colocación de los instrumentos. Incluye el anclaje de los brazos robóticos a los trocares.

Desde que el cirujano se sienta para iniciar el procedimiento hasta que se para al final de éste. 
Tabla II

EXPERIENCIAS REPORTADAS EN LA LITERATURA UTILIZANDO EL MONTAJE LATERAL

\begin{tabular}{|c|c|c|c|c|c|c|}
\hline Autor & Año & $\begin{array}{l}\text { Pacientes } \\
\text { (n) }\end{array}$ & Cirugías & $\begin{array}{l}\text { Tiempo de } \\
\text { montaje } \\
\text { (min) }\end{array}$ & $\begin{array}{l}\text { Tiempo de } \\
\text { consola } \\
\text { (min) }\end{array}$ & Comentarios \\
\hline $\begin{array}{l}\text { Uffort y } \\
\text { cols (4) }\end{array}$ & 2011 & 50 & Prostatectomía radical & 5,5 & 180 & $\begin{array}{l}\text { Tiempo instalación } \\
\text { más corto que montaje } \\
\text { central. Tiempo } \\
\text { montaje similar. Tiempo } \\
\text { operatorio más largo que } \\
\text { montaje central. }\end{array}$ \\
\hline $\begin{array}{l}\text { Woods y } \\
\text { cols (5) }\end{array}$ & 2011 & 12 & $\begin{array}{l}\text { Histerectomía simple y } \\
\text { radical }\end{array}$ & 14,9 & 240 & $\begin{array}{l}\text { Tiempo montaje mayor } \\
\text { con montaje lateral, } \\
\text { no significativo. Mayor } \\
\text { facilidad en extracción } \\
\text { útero }>400 \mathrm{~g}\end{array}$ \\
\hline $\begin{array}{l}\text { Jacob y } \\
\text { cols (13) }\end{array}$ & 2011 & 5 & $\begin{array}{l}\text { Linfadenectomía } \\
\text { lumbo-aórtica }\end{array}$ & ND & 213 & \\
\hline $\begin{array}{l}\text { Hellan y } \\
\text { cols (14) }\end{array}$ & 2009 & 4 & Resección anterior baja & ND & ND & $\begin{array}{l}\text { Dos disecciones } \\
\text { cadavéricas. }\end{array}$ \\
\hline $\begin{array}{l}\text { Choi y } \\
\text { cols (15) }\end{array}$ & 2009 & 50 & Resección anterior baja & ND & 305 & $\begin{array}{l}\text { No se requiere } \\
\text { desmontar el robot } \\
\text { para movilizar } \\
\text { flexura esplénica y } \\
\text { permite la evaluación } \\
\text { colonoscópica } \\
\text { intraoperatoria }\end{array}$ \\
\hline
\end{tabular}

Con respecto al tiempo de trocares, al comparar nuestros primeros 10 casos con los posteriores, no observamos diferencias significativas $(p=0,7)$. El tiempo de montaje fue significativamente más corto en los últimos 12 casos $(p=0,03)$.

Dos pacientes experimentaron complicaciones perioperatorias: una lesión vesical (reparada durante la misma cirugía), y una perforación intestinal diagnosticada al 6 o día postoperatorio que requirió laparotomía y una derivación digestiva. Ninguna paciente requirió transfusión sanguínea. La estadía hospitalaria media fue de 36 horas (rango: 18-96 horas).

\section{DISCUSIÓN}

Desde que el sistema quirúrgico da Vinci fue aprobado por la FDA en 2005 para su utilización en cirugía ginecológica, la mayoría de las publicaciones al respecto han reportado sus resultados utilizando el montaje clásico o central docking $(1,2,8)$. Sin embargo, aunque este tipo de montaje ha demostrado su éxito en otras especialidades (9), su uso en ginecología limita en forma importante el acceso vaginal $(2,10)$. Al igual que en la cirugía laparoscópica tradicional (3), en la cirugía robótica el segundo asistente (situado entre las piernas de la paciente) es fundamental para la exposición y extracción de especímenes, sobre todo en procedimientos más complejos como la histerectomía, la promonto fijación, o la cirugía de endometriosis (6).

Así, con el fin de superar esta importante dificultad decidimos utilizar el montaje lateral en todas nuestras pacientes, desde el inicio de la curva de aprendizaje de la cirugía robótica ginecológica en Clínica Santa María. Además, algunos estudios han reportado la necesidad de realizar más de un montaje, por ejemplo, para acceder adecuadamente al territorio lumbo-aórtico durante la estadificación de cánceres ginecológicos $(12,13)$. Esta limitante puede ser obviada con el uso del montaje lateral (Tabla III). Sólo un estudio ha descrito el montaje lateral utilizando un robot de primera generación (5), comparándolo con una cohorte histórica de pacientes similares en que se utilizó el montaje central; estos 
Tabla III

PROCEDIMIENTOS QUIRÚRGICOS EFECTUADOS CON CIRUGÍA ROBÓTICA

\begin{tabular}{lc}
\hline Procedimiento & $\mathrm{n}$ \\
\hline Histerectomía (con o sin SOB) & 18 \\
Linfadenectomía pelviana & 4 \\
Anexectomía & 1 \\
Omentectomía & 1 \\
Resección endometriosis & 1 \\
Linfadenectomía lumbo-aórtica (muestreo) & 1 \\
Promontofijación & 1 \\
Miomectomía & 1 \\
\hline
\end{tabular}

SOB: salpingo ooforectomía bilateral.

autores reportan una evidente ventaja del montaje lateral, en términos de acceso y tiempo quirúrgico. Adicionalmente, postulan que dichas ventajas pudieran traducirse en una disminución de los costos asociados (5). La factibilidad del montaje lateral se debe probablemente a la mayor versatilidad y flexibilidad que ofrecen los robots de segunda generación; estos incorporan instrumentos más largos y mayor alcance de brazos, facilitando el acceso a todos los cuadrantes del abdomen en un solo montaje (single docking) (13-15). En nuestra experiencia, si bien no contamos con un grupo control, el hecho de haber utilizado el montaje lateral desde el inicio del programa de cirugía robótica nos permitió una adopción rápida de la técnica, con tiempos de montaje comparables con los reportados en la literatura, tanto para el montaje central (2), como lateral (5). Cuando estos tiempos se estratifican de acuerdo al número de casos, nuestros resultados siguen siendo favorables (2). Si bien, la existencia de una curva de aprendizaje para el montaje e instalación del robot está bien documentada $(16,17)$, ésta no ha sido determinada para el montaje lateral. Nuestros resultados sugieren que esta curva podría ser comparable con las curvas de aprendizaje descritas para el montaje clásico $(2,17)$.

Respecto al tiempo de consola, nuestros resultados se comparan con los reportados en la literatura, sin embargo, es importante destacar que los tiempos quirúrgicos varían lógicamente dependiendo de la complejidad del tipo de cirugía. En esta serie, como en otras (16), la cirugía más frecuente fue la histerectomía. Esto se debe, probablemente, a que esta es un procedimiento robótico bien estandarizado (8). En esta serie, el tiempo medio de consola para la histerectomía fue de 101 minutos y es comparable con estudios previos en los que se analizan la factibilidad, la técnica y los resultados de la histerectomía robótica $(8,17)$. Al considerar toda la serie, el tiempo de consola es comparable con los resultados de estudios previos $(1,2,8)$.

En nuestro estudio la tasa de complicaciones fue de $9 \%(2 / 22)$, lo que si bien es mayor a lo reportado por grupos experimentados $(3,8,10)$, es comparable a lo descrito por otros estudios que evalúan las curvas de aprendizaje en cirugía robótica ginecológica $(2,8)$. Sin embargo, es importante destacar que ninguna de estas complicaciones es atribuible al uso del montaje lateral. En este sentido, es importante mencionar que hasta ahora, no hemos notado ninguna interferencia de esta nueva forma de montaje en el desarrollo de la cirugía robótica ginecológica. En tres cirugías ocurrió una colisión de algunos de los brazos robóticos. Esta situación, aunque infrecuente, puede ocurrir con el uso del montaje lateral (4) y parece estar más relacionada con la distancia entre los trocares que con el montaje del robot. Esto es especialmente cierto en pacientes pequeñas o delgadas (15).

La cirugía robótica es un avance en el desarrollo de la cirugía mínimamente invasiva. Aporta una mejor visión del campo operatorio y una mayor precisión de movimiento, dado el uso de instrumental articulado. La limitante mayor que tienen los equipos de segunda generación es el no contar con sensación de tacto, lo cual es lo más complejo de incorporar en la curva de aprendizaje. A pesar de esto, la curva de aprendizaje de la cirugía robótica parece requerir de un menor número de casos comparada con la misma curva de la cirugía laparoscópica tradicional.

\section{CONCLUSIÓN}

Elmontaje lateral esfácil deadoptary proporciona un acceso vaginal más adecuado, facilitando la exposición y la extracción de especímenes durante la cirugía robótica ginecológica.

\section{REFERENCIAS}

1. Advincula AP, Wang K. Evolving role and current state of robotics in minimally invasive gynaecologic surgery. J Minim Invasive Gynecol 2009;16:291-301.

2. Marengo F, Larraín D, Babilonti L, Spinillo A. Learning experience using the double-console Da Vinci surgical system in gynecology: a prospective cohort study in a University hospital. Arch Gynecol Obstet 2012;285: 441-5.

3. Velemir L, Azuar AS, Botchorishvili R, Canis M, Jardon $\mathrm{K}$, Rabischong $\mathrm{B}$, et al. Optimizing the role of surgeon assistants during a laparoscopic hysterectomy. Gynecol Obstet Fertil 2009;37:74-80. 
4. Uffort EE, Jensen JC. Side docking the robot for robotic laparoscopic radical prostatectomy. JSLS 2011;15:200-2.

5. Woods DL, Hou JY, Riemers L, Gupta D, Kuo DY. Side-docking in robotic-assisted gynaecologic cancer surgery. Int J Med Robot 2011;7:51-4.

6. Einarsson JI, Hibner M, Advincula AP. Sidedocking: an alternative docking method for gynaecologic robotic surgery. Rev Obstet Gynecol 2011;4:123-5.

7. Botchorishvili R. Installation et mise en place de la coelioscopie. En: Mage G, Botchorishvili R, Canis M, Jardon K, Manhes H, Pouly JL, Rabischong B, Wattiez A (eds). Chirurgie coelio scopique en gynécologie. Issy-les-Moulineaux: Elsevier-Masson 2007;121-54.

8. Reynolds RK, Advincula AP. Robot-assisted laparoscopic hysterectomy: technique and initial experience. Am J Surg 2006;191:555-60.

9. Tewari A, Peabody J, Sarle R, Balakrishnan G, Hemal A, Shrivastava A, et al. Technique of Da Vinci robotassisted anatomic radical prostatectomy. Urology 2002;60:569-72.

10. Göcmen A, Sanlikan F, Ucar MG. Turkey`s experience of robotic-assisted laparoscopic hysterectomy: a series of 25 consecutive cases. Arch Gynecol Obstet 2010;282:163-71.

11. Field JB, Benoit MF, Dinh TA, Diaz-Arrastia C.
Computer-enhanced robotic surgery in gynaecologic oncology. Surg Endosc 2007;21:244-6.

12. Magrina JF, Long JB, Kho RM, Giles DL, Montero RP, Magtibay PM. Robotic transperitoneal infrarrenal aortic lymphadenectomy: technique and results. Int $\mathrm{J}$ Gynecol Cancer 2010;20:184-7.

13. Jacob KA, Zanagnolo V, Magrina JF, Magtibay PM. Robotic transperitoneal infrarenal aortic lymphadenectomy for gynaecologic malignancy: a left lateral approach. J Laparoendosc Adv SurgTech A 2011;21:733-6.

14. Hellan M, Stein H, Pigazzi A. Totally robotic low anterior resection with total mesorectal excision and splenic flexure mobilization. Surg Endosc 2009;23;447-51.

15. Choi DJ, Kim SH, Lee PJ, Kim J, Woo SU. Single-stage totally robotic dissection for rectal cancer surgery: technique and short-term out come in 50 consecutive patients. Dis Colon Rectum 2009;52:1824-30.

16. Lenihan JP Jr, Kovanda C, Seshandri-Kreaden $U$. What is the learning curve for robotic assisted gynaecologic surgery? J Minim Invasive Gynecol 2008;15:589-94.

17. Kho RM, Hilger WS, Hentz JG, Magtibay PM, Magrina JF. Robotic hysterectomy: technique and initial outcomes. Am J Obstet Gynecol 2007;197:1131 e1-4. 